\title{
ON HERMITE-HADAMARD TYPE INEQUALITIES FOR RIEMANN-LIOUVILLE FRACTIONAL INTEGRALS
}

\author{
MEHMET ZEKI SARIKAYA AND HÜSEYIN YILDIRIM
}

Received 07 April, 2014

\begin{abstract}
In this paper, we have established Hermite-Hadamard-type inequalities for fractional integrals and will be given an identity. With the help of this fractional-type integral identity, we give some integral inequalities connected with the left-side of Hermite-Hadamard-type inequalities for Riemann-Liouville fractional integrals.
\end{abstract}

2010 Mathematics Subject Classification: 26D07; 26D10; 26D15; 26A33

Keywords: Hermite-Hadamard's inequalities, Riemann-Liouville fractional integral, integral inequalities.

\section{INTRODUCTION}

The inequalities discovered by C. Hermite and J. Hadamard for convex functions are very important in the literature (see, e.g.,[20, p.137], [10]). These inequalities state that if $f: I \rightarrow \mathbb{R}$ is a convex function on the interval $I$ of real numbers and $a, b \in I$ with $a<b$, then

$$
f\left(\frac{a+b}{2}\right) \leq \frac{1}{b-a} \int_{a}^{b} f(x) d x \leq \frac{f(a)+f(b)}{2} .
$$

Both inequalities hold in the reversed direction if $f$ is concave. We note that Hadamard's inequality may be regarded as a refinement of the concept of convexity and it follows easily from Jensen's inequality. Hadamard's inequality for convex functions has received renewed attention in recent years and a remarkable variety of refinements and generalizations have been found (see, for example, $[1-4,10-13,15-17,19$, 20,26,27]) and the references cited therein.

The classical Hermite-Hadamard inequality provides estimates of the mean value of a continuous convex function $f:[a, b] \rightarrow \mathbb{R}$.

Definition 1. The function $f:[a, b] \subset \mathbb{R} \rightarrow \mathbb{R}$, is said to be convex if the following inequality holds

$$
f(\lambda x+(1-\lambda) y) \leq \lambda f(x)+(1-\lambda) f(y)
$$

for all $x, y \in[a, b]$ and $\lambda \in[0,1]$. We say that $f$ is concave if $(-f)$ is convex. 
In [15] in order to prove some inequalities related to Hadamard's inequality Kırmac1 used the following lemma:

Lemma 1. Let $f: I^{\circ} \subset \mathbb{R} \rightarrow \mathbb{R}$, be a differentiable mapping on $I^{\circ}, a, b \in I^{\circ}\left(I^{\circ}\right.$ is the interior of I) with $a<b$. If $f^{\prime} \in L([a, b])$, then we have

$$
\begin{aligned}
& \frac{1}{b-a} \int_{a}^{b} f(x) d x-f\left(\frac{a+b}{2}\right) \\
& \quad=(b-a)\left[\int_{0}^{\frac{1}{2}} t f^{\prime}(t a+(1-t) b) d t+\int_{\frac{1}{2}}^{1}(t-1) f^{\prime}(t a+(1-t) b) d t\right] .
\end{aligned}
$$

Also, in [15], Kurmacı obtained the following inequalities for differentiable mappings which are connected with Hermite-Hadamard's inequality:

Theorem 1. Let $f: I^{\circ} \subset \mathbb{R} \rightarrow \mathbb{R}$ be a differentiable mapping on $I^{\circ}, a, b \in I^{\circ}$ with $a<b$. If the mapping $\left|f^{\prime}\right|$ is convex on $[a, b]$, then we have

$$
\left|\frac{1}{b-a} \int_{a}^{b} f(x) d x-f\left(\frac{a+b}{2}\right)\right| \leq \frac{b-a}{8}\left(\left|f^{\prime}(a)\right|+\left|f^{\prime}(b)\right|\right) .
$$

Theorem 2. Let $f: I^{\circ} \subset \mathbb{R} \rightarrow \mathbb{R}$, be a differentiable mapping on $I^{\circ}, a, b \in I^{\circ}$ with $a<b$ and $p>1$. If the mapping $\left|f^{\prime}\right|^{\frac{p}{p-1}}$ is convex on $[a, b]$, then

$$
\begin{aligned}
& \left|\frac{1}{b-a} \int_{a}^{b} f(x) d x-f\left(\frac{a+b}{2}\right)\right| \leq \\
& \leq \frac{(b-a)}{4}\left(\frac{1}{p+1}\right)^{\frac{1}{p}}\left\{\left(\left|f^{\prime}(a)\right|^{\frac{p}{p-1}}+3\left|f^{\prime}(b)\right|^{\frac{p}{p-1}}\right)^{\frac{p-1}{p}}\right. \\
& \left.\quad+\left(3\left|f^{\prime}(a)\right|^{\frac{p}{p-1}}+\left|f^{\prime}(b)\right|^{\frac{p}{p-1}}\right)^{\frac{p-1}{p}}\right\} \\
& \leq \frac{b-a}{4}\left(\frac{4}{p+1}\right)^{\frac{1}{p}}\left[\left|f^{\prime}(a)\right|+\left|f^{\prime}(b)\right|\right] .
\end{aligned}
$$

Meanwhile, Sarikaya et al.[25] presented the following important integral identity including the first-order derivative of $f$ to establish many interesting HermiteHadamard type inequalities for convexity functions via Riemann-Liouville fractional integrals of the order $\alpha>0$.

Lemma 2. Let $f:[a, b] \rightarrow \mathbb{R}$ be a differentiable mapping on $(a, b)$ with $a<b$. If $f^{\prime} \in L[a, b]$, then the following equality for fractional integrals holds:

$$
\frac{f(a)+f(b)}{2}-\frac{\Gamma(\alpha+1)}{2(b-a)^{\alpha}}\left[J_{a+}^{\alpha} f(b)+J_{b-}^{\alpha} f(a)\right]
$$




$$
=\frac{b-a}{2} \int_{0}^{1}\left[(1-t)^{\alpha}-t^{\alpha}\right] f^{\prime}(t a+(1-t) b) d t .
$$

It is remarkable that Sarikaya et al.[25] first give the following interesting integral inequalities of Hermite-Hadamard type involving Riemann-Liouville fractional integrals.

Theorem 3. Let $f:[a, b] \rightarrow \mathbb{R}$ be a positive function with $0 \leq a<b$ and $f \in$ $L_{1}[a, b]$. If $f$ is a convex function on $[a, b]$, then the following inequalities for fractional integrals hold:

$$
f\left(\frac{a+b}{2}\right) \leq \frac{\Gamma(\alpha+1)}{2(b-a)^{\alpha}}\left[J_{a+}^{\alpha} f(b)+J_{b-}^{\alpha} f(a)\right] \leq \frac{f(a)+f(b)}{2}
$$

with $\alpha>0$.

In the following we will give some necessary definitions and mathematical preliminaries of fractional calculus theory which are used further in this paper. More details, one can consult $[14,18,21]$.

Definition 2. Let $f \in L_{1}[a, b]$. The Riemann-Liouville integrals $J_{a+}^{\alpha} f$ and $J_{b-}^{\alpha} f$ of order $\alpha>0$ with $a \geq 0$ are defined by

$$
J_{a+}^{\alpha} f(x)=\frac{1}{\Gamma(\alpha)} \int_{a}^{x}(x-t)^{\alpha-1} f(t) d t, x>a
$$

and

$$
J_{b-}^{\alpha} f(x)=\frac{1}{\Gamma(\alpha)} \int_{x}^{b}(t-x)^{\alpha-1} f(t) d t, \quad x<b
$$

respectively. Here, $\Gamma(\alpha)$ is the Gamma function and $J_{a+}^{0} f(x)=J_{b-}^{0} f(x)=f(x)$.

For some recent results connected with fractional integral inequalities see ([5-9, 22-25, 28]).

The aim of this paper is to establish Hermite-Hadamard's inequalities for RiemannLiouville fractional integral similar to the method in [25] and we will investigate some integral inequalities connected with the left hand side of the Hermite-Hadamard type inequalities for fractional integrals.

\section{HERMITE-HADAMARD'S INEQUALITIES FOR FRACTIONAL INTEGRALS}

Hermite-Hadamard's inequalities can be represented in fractional integral forms as follows: 
Theorem 4. Let $f:[a, b] \rightarrow \mathbb{R}$ be a positive function with $0 \leq a<b$ and $f \in$ $L_{1}[a, b]$. If $f$ is a convex function on $[a, b]$, then the following inequalities for fractional integrals hold:

$$
f\left(\frac{a+b}{2}\right) \leq \frac{2^{\alpha-1} \Gamma(\alpha+1)}{(b-a)^{\alpha}}\left[J_{\left(\frac{a+b}{2}\right)+}^{\alpha} f(b)+J_{\left(\frac{a+b}{2}\right)-}^{\alpha} f(a)\right] \leq \frac{f(a)+f(b)}{2}
$$

with $\alpha>0$.

Proof. Since $f$ is a convex function on $[a, b]$, we have for $x, y \in[a, b]$ with $\lambda=\frac{1}{2}$

$$
f\left(\frac{x+y}{2}\right) \leq \frac{f(x)+f(y)}{2}
$$

i.e., with $x=\frac{t}{2} a+\frac{2-t}{2} b, y=\frac{2-t}{2} a+\frac{t}{2} b$,

$$
2 f\left(\frac{a+b}{2}\right) \leq f\left(\frac{t}{2} a+\frac{2-t}{2} b\right)+f\left(\frac{2-t}{2} a+\frac{t}{2} b\right) .
$$

Multiplying both sides of (2.3) by $t^{\alpha-1}$, then integrating the resulting inequality with respest to $t$ over $[0,1]$, we obtain

$$
\begin{aligned}
& \frac{2}{\alpha} f\left(\frac{a+b}{2}\right) \\
& \leq \int_{0}^{1} t^{\alpha-1} f\left(\frac{t}{2} a+\frac{2-t}{2} b\right) d t+\int_{0}^{1} t^{\alpha-1} f\left(\frac{2-t}{2} a+\frac{t}{2} b\right) d t \\
& =\int_{b}^{\frac{a+b}{2}}\left(\frac{2}{b-a}(b-u)\right)^{\alpha-1} f(u) \frac{2 d u}{a-b}+\int_{a}^{\frac{a+b}{2}}\left(\frac{2}{b-a}(v-a)\right)^{\alpha-1} f(v) \frac{2 d v}{b-a} \\
& =\frac{2^{\alpha} \Gamma(\alpha)}{(b-a)^{\alpha}}\left[J_{\left(\frac{a+b}{2}\right)+}^{\alpha} f(b)+J_{\left(\frac{a+b}{2}\right)-}^{\alpha} f(a)\right]
\end{aligned}
$$

i.e.

$$
f\left(\frac{a+b}{2}\right) \leq \frac{2^{\alpha-1} \Gamma(\alpha+1)}{(b-a)^{\alpha}}\left[J_{\left(\frac{a+b}{2}\right)+}^{\alpha} f(b)+J_{\left(\frac{a+b}{2}\right)-}^{\alpha} f(a)\right]
$$

and the first inequality is proved.

For the proof of the second inequality in (2.2) we first note that if $f$ is a convex function, then, for $\lambda \in[0,1]$, it yields

$$
f\left(\frac{t}{2} a+\frac{2-t}{2} b\right) \leq \frac{t}{2} f(a)+\frac{2-t}{2} f(b)
$$

and

$$
f\left(\frac{2-t}{2} a+\frac{t}{2} b\right) \leq \frac{2-t}{2} f(a)+\frac{t}{2} f(b) .
$$


By adding these inequalities we have

$$
f\left(\frac{t}{2} a+\frac{2-t}{2} b\right)+f\left(\frac{2-t}{2} a+\frac{t}{2} b\right) \leq f(a)+f(b) .
$$

Then multiplying both sides of (2.4) by $t^{\alpha-1}$ and integrating the resulting inequality with respest to $t$ over $[0,1]$, we obtain

$$
\begin{aligned}
& \int_{0}^{1} t^{\alpha-1} f\left(\frac{t}{2} a+\frac{2-t}{2} b\right) d t+\int_{0}^{1} t^{\alpha-1} f\left(\frac{2-t}{2} a+\frac{t}{2} b\right) d t \\
& \leq[f(a)+f(b)] \int_{0}^{1} t^{\alpha-1} d t
\end{aligned}
$$

i.e.

$$
\frac{2^{\alpha} \Gamma(\alpha)}{(b-a)^{\alpha}}\left[J_{\left(\frac{a+b}{2}\right)+}^{\alpha} f(b)+J_{\left(\frac{a+b}{2}\right)-}^{\alpha} f(a)\right] \leq \frac{f(a)+f(b)}{\alpha} .
$$

The proof is completed.

Remark 1. If in Theorem 4, we let $\alpha=1$, then the inequalities (2.1) become the inequalities (1.1).

\section{FRACTIONAL INEQUALITIES FOR CONVEX FUNCTIONS}

We need the following lemma. With the help of this, we give some integral inequalities connected with the left-side of Hermite-Hadamard-type inequalities for Riemann-Liouville fractional integrals.

Lemma 3. Let $f:[a, b] \rightarrow \mathbb{R}$ be a differentiable mapping on $(a, b)$ with $a<b$. If $f^{\prime} \in L[a, b]$, then the following equality for fractional integrals holds:

$$
\begin{aligned}
& \frac{2^{\alpha-1} \Gamma(\alpha+1)}{(b-a)^{\alpha}}\left[J_{\left(\frac{a+b}{2}\right)+}^{\alpha} f(b)+J_{\left(\frac{a+b}{2}\right)-}^{\alpha} f(a)\right]-f\left(\frac{a+b}{2}\right) \\
= & \frac{b-a}{4}\left\{\int_{0}^{1} t^{\alpha} f^{\prime}\left(\frac{t}{2} a+\frac{2-t}{2} b\right) d t-\int_{0}^{1} t^{\alpha} f^{\prime}\left(\frac{2-t}{2} a+\frac{t}{2} b\right) d t\right\}
\end{aligned}
$$

with $\alpha>0$. 
Proof. Integrating by parts

$$
\begin{aligned}
I_{1} & =\int_{0}^{1} t^{\alpha} f^{\prime}\left(\frac{t}{2} a+\frac{2-t}{2} b\right) d t \\
& =\left.t^{\alpha} \frac{2}{a-b} f\left(\frac{t}{2} a+\frac{2-t}{2} b\right)\right|_{0} ^{1}-\int_{0}^{1} \alpha t^{\alpha-1} f\left(\frac{t}{2} a+\frac{2-t}{2} b\right) \frac{2}{a-b} d t \\
& =-\frac{2}{b-a} f\left(\frac{a+b}{2}\right)-\frac{2 \alpha}{a-b} \int_{b}^{\frac{a+b}{2}}\left(\frac{2}{b-a}(b-x)\right)^{\alpha-1} \frac{2}{a-b} f(x) d x \\
& =-\frac{2}{b-a} f\left(\frac{a+b}{2}\right)+\frac{2^{\alpha+1} \Gamma(\alpha+1)}{(b-a)^{\alpha+1}} J_{\left(\frac{a+b}{2}\right)-}^{\alpha} f(b)
\end{aligned}
$$

and similarly we get,

$$
\begin{aligned}
I_{2} & =\int_{0}^{1} t^{\alpha} f^{\prime}\left(\frac{2-t}{2} a+\frac{t}{2} b\right) d t \\
& =\left.t^{\alpha} \frac{2}{b-a} f\left(\frac{2-t}{2} a+\frac{t}{2} b\right)\right|_{0} ^{1}-\frac{2 \alpha}{b-a} \int_{0}^{1} t^{\alpha-1} f\left(\frac{2-t}{2} a+\frac{t}{2} b\right) d t \\
& =\frac{2}{b-a} f\left(\frac{a+b}{2}\right)-\frac{2 \alpha}{b-a} \int_{a}^{\frac{a+b}{2}}\left(\frac{2}{b-a}(x-a)\right)^{\alpha-1} f(x) \frac{2}{b-a} d x \\
& =\frac{2}{b-a} f\left(\frac{a+b}{2}\right)-\frac{2^{\alpha+1} \Gamma(\alpha+1)}{(b-a)^{\alpha+1}} J_{\left(\frac{a+b}{2}\right)+}^{\alpha} f(a) .
\end{aligned}
$$

By using (3.2) and (3.3), it follows that

$$
I_{1}-I_{2}=-\frac{4}{b-a} f\left(\frac{a+b}{2}\right)+\frac{2^{\alpha+1} \Gamma(\alpha+1)}{(b-a)^{\alpha+1}}\left[J_{\left(\frac{a+b}{2}\right)+}^{\alpha} f(b)+J_{\left(\frac{a+b}{2}\right)-}^{\alpha} f(a)\right] \text {. }
$$

Thus, by multiplying the both sides by $\frac{b-a}{4}$, we have the conclusion (3.1).

Corollary 1. If in Lemma 3, we let $\alpha=1$, then the equality (3.1) becomes the following equality

$$
\begin{aligned}
& \frac{1}{b-a} \int_{a}^{b} f(x) d x-f\left(\frac{a+b}{2}\right) \\
& =\frac{b-a}{4}\left\{\int_{0}^{1} t f^{\prime}\left(\frac{t}{2} a+\frac{2-t}{2} b\right) d t-\int_{0}^{1} t f^{\prime}\left(\frac{2-t}{2} a+\frac{t}{2} b\right) d t\right\} .
\end{aligned}
$$

Using this Lemma 3, we can obtain the following fractional integral inequality:

Theorem 5. Let $f:[a, b] \rightarrow \mathbb{R}$ be a differentiable mapping on $(a, b)$ with $a<b$. If $\left|f^{\prime}\right|^{q}$ is convex on $[a, b]$ for $q \geq 1$, then the following inequality for fractional 
integrals holds:

$$
\begin{aligned}
& \left|\frac{2^{\alpha-1} \Gamma(\alpha+1)}{(b-a)^{\alpha}}\left[J_{\left(\frac{a+b}{2}\right)+}^{\alpha} f(b)+J_{\left(\frac{a+b}{2}\right)-}^{\alpha} f(a)\right]-f\left(\frac{a+b}{2}\right)\right| \\
& \leq \frac{b-a}{4(\alpha+1)}\left(\frac{1}{2(\alpha+2)}\right)^{\frac{1}{q}}\left\{\left((\alpha+1)\left|f^{\prime}(a)\right|^{q}+(\alpha+3)\left|f^{\prime}(b)\right|^{q}\right)^{\frac{1}{q}}\right. \\
& \left.\quad+\left((\alpha+3)\left|f^{\prime}(a)\right|^{q}+(\alpha+1)\left|f^{\prime}(b)\right|^{q}\right)^{\frac{1}{q}}\right\}
\end{aligned}
$$

Proof. Firstly, we suppose that $q=1$. Using Lemma 3 and the convexity of $\left|f^{\prime}\right|$, we find

$$
\begin{aligned}
& \left|\frac{2^{\alpha-1} \Gamma(\alpha+1)}{(b-a)^{\alpha}}\left[J_{\left(\frac{a+b}{2}\right)+}^{\alpha} f(b)+J_{\left(\frac{a+b}{2}\right)-}^{\alpha} f(a)\right]-f\left(\frac{a+b}{2}\right)\right| \\
& \leq \frac{b-a}{4} \int_{0}^{1} t^{\alpha}\left\{\left|f^{\prime}\left(\frac{t}{2} a+\frac{2-t}{2} b\right)\right|+\left|f^{\prime}\left(\frac{2-t}{2} a+\frac{t}{2} b\right)\right|\right\} d t \\
& \left.\left.\leq \frac{b-a}{4}\left[\mid f^{\prime}(a)\right)|+| f^{\prime}(b)\right) \mid\right] \int_{0}^{1} t^{\alpha} d t \\
& \left.\left.=\frac{b-a}{4(\alpha+1)}\left[\mid f^{\prime}(a)\right)|+| f^{\prime}(b)\right) \mid\right] .
\end{aligned}
$$

Secondly, we suppose that $q>1$. Using Lemma 3 and the power mean inequality, and the convexity of $\left|f^{\prime}\right|^{q}$, we find

$$
\begin{aligned}
& \left|\frac{2^{\alpha-1} \Gamma(\alpha+1)}{(b-a)^{\alpha}}\left[J_{\left(\frac{a+b}{2}\right)+}^{\alpha} f(b)+J_{\left(\frac{a+b}{2}\right)-}^{\alpha} f(a)\right]-f\left(\frac{a+b}{2}\right)\right| \\
& \leq \frac{b-a}{4}\left\{\left(\int_{0}^{1} t^{\alpha}\left|f^{\prime}\left(\frac{t}{2} a+\frac{2-t}{2} b\right)\right|^{q} d t\right)^{\frac{1}{q}}\right. \\
& \left.\quad+\left(\int_{0}^{1} t^{\alpha}\left|f^{\prime}\left(\frac{2-t}{2} a+\frac{t}{2} b\right)\right|^{q} d t\right)^{\frac{1}{q}}\right\} \\
& \leq \frac{b-a}{4} \frac{1}{(\alpha+1)^{\frac{1}{p}}}\left\{\left(\int_{0}^{1}\left[\frac{t^{\alpha+1}}{2}\left|f^{\prime}(a)\right|^{q}+\frac{2 t^{\alpha}-t^{\alpha+1}}{2}\left|f^{\prime}(b)\right|^{q}\right] d t\right)^{\frac{1}{q}}\right. \\
& \left.\quad+\left(\int_{0}^{1}\left[\frac{2 t^{\alpha}-t^{\alpha+1}}{2}\left|f^{\prime}(a)\right|^{q}+\frac{t^{\alpha+1}}{2}\left|f^{\prime}(b)\right|^{q}\right] d t\right)^{\frac{1}{q}}\right\}
\end{aligned}
$$




$$
\begin{aligned}
= & \frac{b-a}{4} \frac{1}{(\alpha+1)^{\frac{1}{p}}}\left\{\left(\frac{1}{2(\alpha+2)}\left|f^{\prime}(a)\right|^{q}+\frac{(\alpha+3)}{2(\alpha+1)(\alpha+2)}\left|f^{\prime}(b)\right|^{q}\right)^{\frac{1}{q}}\right. \\
& \left.+\left(\frac{(\alpha+3)}{2(\alpha+1)(\alpha+2)}\left|f^{\prime}(a)\right|^{q}+\frac{1}{2(\alpha+2)}\left|f^{\prime}(b)\right|^{q}\right)^{\frac{1}{q}}\right\} .
\end{aligned}
$$

The proof of Theorem 5 is complete.

Remark 2. If we take $\alpha=1$ and $q=1$ in Theorem 5, then the inequality (3.5) becomes the inequality (1.2) of Theorem 1 .

Theorem 6. Let $f:[a, b] \rightarrow \mathbb{R}$ be a differentiable mapping on $(a, b)$ with $a<b$. If $\left|f^{\prime}\right|^{q}$ is convex on $[a, b]$ for $q>1$, then the following inequality for fractional integrals holds:

$$
\begin{aligned}
& \left|\frac{2^{\alpha-1} \Gamma(\alpha+1)}{(b-a)^{\alpha}}\left[J_{\left(\frac{a+b}{2}\right)+}^{\alpha} f(b)+J_{\left(\frac{a+b}{2}\right)-}^{\alpha} f(a)\right]-f\left(\frac{a+b}{2}\right)\right| \\
& \leq \frac{b-a}{4}\left(\frac{1}{\alpha p+1}\right)^{\frac{1}{p}}\left[\left(\frac{\left.\left.\mid f^{\prime} a\right)|+3| f^{\prime}(b)\right) \mid}{4}\right)^{\frac{1}{q}}+\left(\frac{\left.\left.3 \mid f^{\prime} a\right)|+| f^{\prime}(b)\right) \mid}{4}\right)^{\frac{1}{q}}\right] \\
& \leq \frac{b-a}{4}\left(\frac{4}{\alpha p+1}\right)^{\frac{1}{p}}\left[\left|f^{\prime}(a)\right|+\left|f^{\prime}(b)\right|\right]
\end{aligned}
$$

where $\frac{1}{p}+\frac{1}{q}=1$.

Proof. From Lemma 3, using the well-known Hölder's inequality and the convexity of $\left|f^{\prime}\right|^{q}$, we find

$$
\begin{aligned}
& \left|\frac{2^{\alpha-1} \Gamma(\alpha+1)}{(b-a)^{\alpha}}\left[J_{\left(\frac{a+b}{2}\right)+}^{\alpha} f(b)+J_{\left(\frac{a+b}{2}\right)-}^{\alpha} f(a)\right]-f\left(\frac{a+b}{2}\right)\right| \\
& \leq \frac{b-a}{4}\left(\int_{0}^{1} t^{\alpha p} d t\right)^{\frac{1}{p}}\left\{\left(\int_{0}^{1}\left|f^{\prime}\left(\frac{t}{2} a+\frac{2-t}{2} b\right)\right|^{q} d t\right)^{\frac{1}{q}}\right. \\
& \left.\quad+\left(\int_{0}^{1}\left|f^{\prime}\left(\frac{2-t}{2} a+\frac{t}{2} b\right)\right|^{q} d t\right)^{\frac{1}{q}}\right\} \\
& \leq \frac{b-a}{4}\left(\frac{1}{\alpha p+1}\right)^{\frac{1}{p}}\left\{\left(\left.\int_{0}^{1}\left[\frac{t}{2} \mid f^{\prime}(a)\right)\right|^{q}+\frac{2-t}{2}\left|f^{\prime}(b)\right|^{q}\right] d t\right)^{\frac{1}{q}} \\
& \left.\left.\quad+\left(\left.\int_{0}^{1}\left[\frac{2-t}{2} \mid f^{\prime}(a)\right)\right|^{q}+\frac{t}{2}\left|f^{\prime}(b)\right|^{q}\right] d t\right)^{\frac{1}{q}}\right\}
\end{aligned}
$$




$$
=\frac{b-a}{4}\left(\frac{1}{\alpha p+1}\right)^{\frac{1}{p}}\left[\left(\frac{\left.\left.\mid f^{\prime}(a)\right)|+3| f^{\prime}(b)\right) \mid}{4}\right)^{\frac{1}{q}}+\left(\frac{\left.\left.3 \mid f^{\prime}(a)\right)|+| f^{\prime}(b)\right) \mid}{4}\right)^{\frac{1}{q}}\right] \text {. }
$$

Let $a_{1}=3\left|f^{\prime}(a)\right|^{q}, b_{1}=\left|f^{\prime}(b)\right|^{q}, a_{2}=\left|f^{\prime}(a)\right|^{q}, b_{2}=3\left|f^{\prime}(b)\right|^{q}$. Here, $0<\frac{1}{q}<1$ for $q>1$. Using the fact that,

$$
\sum_{k=1}^{n}\left(a_{k}+b_{k}\right)^{s} \leq \sum_{k=1}^{n} a_{k}^{s}+\sum_{k=1}^{n} b_{k}^{s}
$$

For $(0 \leq s<1), a_{1}, a_{2}, \ldots, a_{n} \geq 0, b_{1}, b_{2}, \ldots, b_{n} \geq 0$, we obtain

$$
\begin{aligned}
& \left|\frac{2^{\alpha-1} \Gamma(\alpha+1)}{(b-a)^{\alpha}}\left[J_{\left(\frac{a+b}{2}\right)+}^{\alpha} f(b)+J_{\left(\frac{a+b}{2}\right)-}^{\alpha} f(a)\right]-f\left(\frac{a+b}{2}\right)\right| \\
& \leq \frac{b-a}{16}\left(\frac{4}{\alpha p+1}\right)^{\frac{1}{p}}\left[\left(3\left|f^{\prime}(a)\right|^{q}+\left|f^{\prime}(b)\right|^{q}\right)^{\frac{1}{q}}+\left(\left|f^{\prime}(a)\right|^{q}+3\left|f^{\prime}(b)\right|^{q}\right)^{\frac{1}{q}}\right] \\
& \leq \frac{b-a}{16}\left(\frac{4}{\alpha p+1}\right)^{\frac{1}{p}}\left(3^{\frac{1}{q}}+1\right)\left[\left|f^{\prime}(a)\right|+\left|f^{\prime}(b)\right|\right] \\
& \leq \frac{b-a}{16}\left(\frac{4}{\alpha p+1}\right)^{\frac{1}{p}} 4\left[\left|f^{\prime}(a)\right|+\left|f^{\prime}(b)\right|\right]
\end{aligned}
$$

which completed proof.

Remark 3. If we take $\alpha=1$ in Theorem 6, then the inequality (3.6) becomes the inequality (1.3) of Theorem 2.

\section{REFERENCES}

[1] M. Alomari and M. Darus, "On the Hadamard's inequality for log-convex functions on the coordinates," Journal of Inequalities and Applications, vol. 2009, p. 13 pages, 2009, doi: 10.1155/2009/283147.

[2] A. Azpeitia, "Convex functions and the Hadamard inequality," Rev. Colombiana Math., vol. 28, pp. 7-12, 1994.

[3] M. K. Bakula and J. Pečarić, "Note on some Hadamard-type inequalities," J. Ineq. Pure and Appl. Math., vol. 5, no. 3, p. Art. 74, 2004.

[4] M. Bakula, M. Özdemir, and J. Pečarić, "Hadamard type inequalities for $m$-convex and $(\alpha, m)$ convex functions," J. Ineq. Pure and Appl. Math., vol. 9, no. 4, p. 96, 2008.

[5] S. Belarbi and Z. Dahmani, "On some new fractional integral inequalities," J. Ineq. Pure and Appl. Math., vol. 10, no. 3, p. Art. 86, 2009.

[6] Z. Dahmani, "New inequalities in fractional integrals," International Journal of Nonlinear Scinece, vol. 9, no. 4, pp. 493-497, 2010.

[7] Z. Dahmani, "On Minkowski and Hermite-Hadamard integral inequalities via fractional integration," Ann. Funct. Anal., vol. 1, no. 1, pp. 51-58, 2010.

[8] Z. Dahmani, L. Tabharit, and S. Taf, "New generalizations of Gruss inequality usin RiemannLiouville fractional integrals," Bull. Math. Anal. Appl., vol. 2, no. 3, pp. 93-99, 2010. 
[9] Z. Dahmani, L. Tabharit, and S. Taf, "Some fractional integral inequalities," Nonl. Sci. Lett. A, vol. 1, no. 2, pp. 155-160, 2010.

[10] S. S. Dragomir and C. E. M. Pearce, "Selected topics on Hermite-Hadamard inequalities and applications," RGMIA Monographs, 2000.

[11] S. Dragomir, "On some new inequalities of Hermite-Hadamard type for $m$-convex functions," Tamkang J. Math., vol. 3, no. 1, 2002.

[12] S. Dragomir and R. Agarwal, "Two inequalities for differentiable mappings and applications to special means of real numbers and to trapezoidal formula," Appl. Math. Lett., vol. 11, no. 5, pp. 91-95, 1998, doi: 10.1016/S0893-9659(98)00086-X.

[13] P. M. Gill, C. E. M. Pearce, and J. Pečarić, "Hadamard's inequality for $r$-convex functions," Journal of Mathematical Analysis and Applications, vol. 215, no. 2, pp. 461-470, 1997, doi: 10.1006/jmaa.1997.5645.

[14] R. Gorenflo and F. Mainardi, "Fractional calculus: integral and differential equations of fractional order," Springer Verlag, Wien, pp. 223-276, 1997.

[15] U. Kirmaci, "Inequalities for differentiable mappings and applications to special means of real numbers to midpoint formula," Appl. Math. Comput., vol. 147, no. 5, pp. 137-146, 2004, doi: 10.1016/S0096-3003(02)00657-4.

[16] U. Kirmaci, M. Bakula, M. Ozdemir, and J. Pecaric, "Hadamard-tpye inequalities for s-convex functions," Appl. Math. Comput., vol. 193, pp. 26-35, 2007, doi: 10.1016/j.amc.2007.03.030.

[17] U. Kirmaci and M. Ozdemir, "On some inequalities for differentiable mappings and applications to special means of real numbers and to midpoint formula," Appl. Math. Comput., vol. 153, pp. 361-368, 2004, doi: 10.1016/S0096-3003(03)00637-4.

[18] S. Miller and B. Ross, "On introduction to the fractional calculus and fractional differential equations," John Wiley \& Sons, USA, vol. 13, p. 2, 1993.

[19] M. Ozdemir, M. Avci, and E. a. Set, "On some inequalities of Hermite-Hadamard type via m-convexity," Applied Mathematics Letter, vol. 23, no. 9, pp. 1065-1070, 2010, doi: 10.1016/j.aml.2010.04.037.

[20] J. Pecaric, F. Proschan, and Y. L. Tong, "Convex functions, partial orderings and statistical applications," Academic Press, Boston, 1992.

[21] I. Podlubni, "Fractional differential equations," Academic Press, San Diego, 1999.

[22] H. Sarikaya, M. Z.and Yaldiz, "On weighted Montogomery identities for Riemann-Liouville fractional integrals," Konuralp Journal of Mathematics, vol. 1, no. 1, pp. 48-53, 2013.

[23] M. Z. Sarikaya, "Ostrowski type inequalities involving the right Caputo fractional derivatives belong to $L_{p}$," Facta Universitatis, Series Mathematics and Informatics, vol. 27, no. 2, pp. 191197, 2012.

[24] M. Z. Sarikaya and H. Ogunmez, "On new inequalities via Riemann-Liouville fractional integration," Abstract and Applied Analysis, vol. 2012, p. 10 pages, 2012, doi: 10.1155/2012/428983.

[25] M. Z. Sarikaya, E. Set, H. Yaldiz, and N. Basak, "Hermite -Hadamard's inequalities for fractional integrals and related fractional inequalities," Mathematical and Computer Modelling, vol. 57, pp. 2403-2407, 2013, doi: 10.1016/j.mcm.2011.12.048.

[26] E. Set, M. O. Ozdemir, and S. S. Dragomir, "On Hadamard-Type inequalities involving several kinds of convexity," Journal of Inequalities and Applications, vol. Article ID 286845, p. 12, 2010, doi: $10.1155 / 2010 / 286845$.

[27] E. Set, M. O. Ozdemir, and S. S. Dragomir, "On the Hermite-Hadamard inequality and other integral inequalities involving two functions," Journal of Inequalities and Applications, vol. Article ID 148102, p. 9, 2010, doi: 10.1155/2010/148102.

[28] J. Wang, X. Li, M. Feckan, and Y. Zhou, "Hermite-Hadamard-type inequalities for RiemannLiouville fractional integrals via two kinds of convexity," Applicable Analysis, pp. 1-13, 2012, doi: 10.1080/00036811.2012.727986. 
Authors' addresses

\section{Mehmet Zeki Sarıkaya}

Department of Mathematics, Faculty of Science and Arts, Düzce University, Düzce-TURKEY

E-mail address: sarikayamz@gmail.com

Hüseyin Yildirim

Department of Mathematics, Faculty of Science and Arts, Kahramanmaraş Sütçü İmam University, Kahramanmaraş, Turkey

E-mail address: hyildir@ksu.edu.tr 